\title{
Identification of Children with Acute Lymphoblastic Leukemia at Low Risk for Tumor Lysis Syndrome
}

\author{
Bahoush $\mathrm{GR}^{1^{*}}$, Yazdi $\mathrm{E}^{2}$, Ansari $\mathrm{SH}^{3}$, Arjmandi $\mathrm{KH}^{3}$ and Vossough $\mathrm{P}^{3}$ \\ ${ }^{1}$ Department of Pediatrics, Pediatric Hematologist and Oncologist, Onco-Pathology Research Center, Ali-Asghar Children Hospital, Iran University of Medical Sciences, \\ Tehran, Iran \\ ${ }^{2}$ General Practitioner, Iran University of Medical Sciences, Tehran, Iran \\ ${ }^{3}$ Department of Pediatrics, Pediatric Hematologist and Oncologist, Ali-Asghar Children Hospital, Iran University of Medical Sciences, Tehran, Iran
}

*Corresponding author: Bahoush GR, Iran University of Medical Sciences, Tehran, Iran, Tel: +989171907100; E-mail: bahoush.gh@iums.ac.ir

Received date: Jul 06, 2015, Accepted date: Oct 29, 2015, Publication date: Nov 03, 2015

Copyright: (c) 2015 Bahoush GR, et al. This is an open-access article distributed under the terms of the Creative Commons Attribution License, which permits unrestricted use, distribution, and reproduction in any medium, provided the original author and source are credited.

\begin{abstract}
Purpose: We determined the prevalence and predictors of TLS in children with acute lymphoblastic leukemia to develop a sensitive prediction rule to identify low risk patients.

Methods: Predictors of TLS were determined in 160 childern with ALL, using univariate and multiple logistic regression analyses.

Results: TLS was diagnosed in 41 cases. Univariate analysis showed splenomegaly, mediastinal mass, T-cell phenotype, central nervous system involvement, lactate dehydrogenase $\geq 2000 \mathrm{U} / \mathrm{L}$, and white blood count (WBC) $\geq 20 \times 10^{9} / \mathrm{L}(\mathrm{P}<0.001)$ were predictors of TLS in these cases. Multiple regression analysis of variables identified CNS and renal involvement, mediastinal mass, and initial WBC $\geq 20 \times 10^{9} / \mathrm{L}$ as independent predictors of TLS.

Conclusions: The above predictors could evaluate the risk of TLS in hematologic malignancies before initiative chemotherapy. Finding a model of independent factors to define a group of ALL children at low risk of TLS could be used to prevent cost of prophylactic treatment modalities.
\end{abstract}

Keywords: Tumor lysis Syndrome (TLS); Acute Lymphoblastic Lymphoma (ALL); Hypocalcemia

\section{Introduction}

Tumor lysis syndrome (TLS) may develop after or before the initiation of conventional therapy in patients with acute lymphoblastic leukemia (ALL) and Burkitt lymphoma. This life threatening syndrome occurs in $5-20 \%$ of cancer patients characterized by hyperuricemia, hyperkalemia, hyperphosphatemia and hypocalcemia, which are the result of massive necrosis or apoptosis of large proliferating tumors, cell lysis and the metabolism of excessive nucleic acids. TLS is clinically defined by the presence of the above laboratory abnormalities and one or more clinical complications such as: renal insufficiency, cardiac arrhythmias/sudden death, and seizure [1].

High tumor growth rate predisposes the patient to develop TLS. Tumors with high sensitivity to chemotherapy and large tumor burden could be important factors of TLS progression in patients with solid tumor Other variables such as elevated serum level of lactate dehydrogenase $(\mathrm{LDH})$,decreased urinary flow rate and older age with impaired renal function may predispose the patient to clinically significant TLS. Furthermore, several studies have been designed to determine high risk patients for TLS but identifying low risk group is equally important so as to avoid unnecessary monitoring and treatment. The aim of this study was describing the risk factors and predictors of TLS in childhood ALL and develop a sensitive model to identify those with lower risk of developing TLS [2-6].

\section{Materials and Methods}

In this cross-sectional analytic study 160 children diagnosed as Acute Lymphoblastic Leukemia (ALL), Tehran (1996-2010) were recruited. Those with incomplete data, partial treatment, patients who did not receive initial ALL therapy, those who were transferred to the other centers within 7 days of admission and Burkitt leukemia were excluded [7-9].

TLS was defined as having two or more of the following criteria:

- hyperurecemia (serum uric acid $>8 \mathrm{mg} / \mathrm{dL}$ )

- Hyperkalemia (Potassium $>5.5 \mathrm{~m} \mathrm{Eq/L}$ )

- hyperphosphatemia (serum phosphorous $>7 \mathrm{mg} / \mathrm{dL}$ )

- hypocalcemia (serum calcium $<8 \mathrm{mg} / \mathrm{dL}$ )

- renal failure (serum creatinin $>1.5$ of normal limits for the age) [10].

WBC and LDH were recorded at admission. Clinical markers of bulky disease were reviewed by physical examination as followings: mediastinal mass on chest radiograph, hepatomegaly (palpable liver $\geq 3$ $\mathrm{cm}$ below the right costal margin), and splenomegaly (palpable spleen $\geq 2 \mathrm{~cm}$ below the left costal margin). Central nervous system (CNS) status and renal involvement (renal enlargement on abdominal imaging studies) were other predictors which were evaluated. A cutoff value of $2000 \mathrm{U} / \mathrm{L}$ was chosen [10] for $\mathrm{LDH}$.

Patients with precursor B-cell ALL received the BFM protocol (conventional/IC BFM 2002) [10]. They received oral prednisolone 30 $\mathrm{mg} / \mathrm{m}^{2} /$ day for the first 7 days plus intrathecal methotrexate on the 
Citation: Bahoush GR, Yazdi E, Ansari SH, Arjmandi KH, Vossough P (2015) Identification of Children with Acute Lymphoblastic Leukemia at Low Risk for Tumor Lysis Syndrome . J Blood Disord Transfus 6: 318. doi:10.4172/2155-9864.1000318

Page 2 of 4

first day (Ref??). Patients with T-cell ALL were treated by LSA2L2/ NY-1 protocol. They received Cyclophosphamide $400 \mathrm{mg} / \mathrm{m}^{2} / \mathrm{d}$ for the first 3 days plus intrathecal cytosine arabinoside which was followed by oral prednisolone $30 \mathrm{mg} / \mathrm{m}^{2} /$ day or dexamethasone $6 \mathrm{mg} / \mathrm{m}^{2} /$ day for 7 days. If there was any TLS (according to clinical/laboratory criteria), patients received $3000 \mathrm{ml} / \mathrm{m}^{2} /$ day Dextrose Saline $1 / 4$ NS with 40 $\mathrm{mEq} / \mathrm{L} \mathrm{NaHCO}_{3}$, and urine output was maintained at $>100 \mathrm{mLlm} / 2 / \mathrm{hr}$ and urine specific gravity <'1.010. $\mathrm{NaHCo}_{3}$ was added to maintain urine $\mathrm{pH}$ at 7-7.5. $\mathrm{NaHCO}_{3}$ was stopped when cytotoxic therapy was initiated or the urine $\mathrm{pH}>7.5$. If TLS was developed, the mentioned fluid therapy was increased to $5000 \mathrm{ml} / \mathrm{m}^{2} /$ day. Due to very high cost of urate oxidase, it was started if there were clinical TLS or initial WBC was more than $50000 / \mu \mathrm{l}$ at presentation. Also, urate oxidase was considered for those patients who could not use allopurinol for any cause.

Univariate analysis and Chi-square were used to evaluate the relationship between variables and the risk of TLS. Those with a pvalue $<0.05$ was considered as predictors of TLS. Those predictors of low risk for TLS with P-value $<0.1$ were entered into the multiple logistic regression analysis by forward logistic regression method.

Study protocol was approved by the local ethical committee and it conforms to the provisions of Declaration of Helsinki (as revised in Tokyo 2004). Patients' anonymity were preserved.

\section{Results}

One hundred and sixty children were included in this study with a mean $( \pm \mathrm{SD})$ age of $5.38( \pm 3.55)$ years (range 7 months to 14 years). Demographic characteristics, clinical and laboratory data of the recruited patients are shown in Table 1.

Among them TLS was presented in 41 (25.6\%). The most common laboratory abnormality in all patients was hypocalcaemia which was observed in 48 cases (30\%). The least frequent laboratory abnormality was hyperphosphatemia (16 cases $=10 \%)$. The most common laboratory abnormality pair for TLS was hypocalcemia and hyperphosphatemia (10 of 160 patients; 6.25\%), followed by concurrent abnormalities of calcium and uric acid (5.6\%).

The results of univariate analysis (Table $2 \mathrm{~A}$ ) showed that CNS and renal involvement and $\mathrm{T}$-cell immunophenotype are among the strongest predictors of TLS. The initial LDH value was a strong predictor of TLS; however, because only $108 \mathrm{LDH}$ samples were determined on the day of admission, this variable could not be included in the multiple regression analysis [11-13]. Also, age at diagnosis and sex were not statistically significant predictor of TLS. Four variables were identified in multiple regression as independent predictors of TLS: mediastinal mass, initial WBC $\geq 20 \times 10^{9} / \mathrm{L}$, CNS and renal involvement at diagnosis (Table 2B).

The absence of all 4 predictors of TLS was used to define a group at low risk of developing TLS (the low-risk TLS group). Of those who fulfilled low-risk TLS criteria $(\mathrm{N}=90), 83$ did not develop TLS, resulting in a negative predictive value of $92.22 \%(95 \% \mathrm{CI}$, $84.81-96.18 \%)$ and a sensitivity of $82.93 \%$ (95\% CI, 68.74-91.47\%).

\begin{tabular}{|l|l|l|}
\hline \multicolumn{2}{|l|}{ Variables } & $\begin{array}{l}\text { Numbers (\%) } \\
\text { N= 160 }\end{array}$ \\
\hline Gender & Male & $91(56.9)$ \\
\cline { 2 - 3 } & Female & $69(43.1)$ \\
\hline Age ( $\geq 10$ years) & & $28(17.5)$ \\
\hline WBC $\geq 20,000 / \mu L$ & & $54(33.75)$ \\
\hline Significant splenomegaly & & $67(41.87)$ \\
\hline Significant hepatomegaly & & $46(28.75)$ \\
\hline Mediastinal mass & & $17(10.62)$ \\
\hline CNS involvement & & $8(5.0)$ \\
\hline Renal involvement & & $15(9.37)$ \\
\hline $\begin{array}{l}\text { Baseline LDH } \\
\text { (N=108) }\end{array}$ & & $27(25)$ \\
\hline ALL immunophenotype & Precursor B-cell & $138(86.2)$ \\
\hline
\end{tabular}

Table 1: Demographic background, clinical and laboratory distribution.

\begin{tabular}{|l|l|l|l|l|l|}
\hline OR & $\mathbf{9 5 \%}$ CI & P-value & TLS $-(\mathbf{n = 1 9 1 )}$ & $\begin{array}{l}\text { TLS }+ \\
(\mathbf{n}=\mathbf{4 1})\end{array}$ & Variables \\
\hline 4.18 & $1.98-8.83$ & $<0.001$ & $30(25.2 \%)$ & $24(58.5 \%)$ & Baseline WBC $\geq 20000 / \mu \mathrm{L}$ \\
\hline 2.38 & $1.36-5.87$ & 0.005 & $42(35.6 \%)$ & $25(61 \%)$ & Splenomegaly \\
\hline 2.54 & $1.2-5.37$ & 0.013 & $28(23.5 \%)$ & $18(43.9 \%)$ & Hepatomegaly \\
\hline 4.45 & $1.56-12.67$ & 0.003 & $8(7.2 \%)$ & $9(25.7 \%)$ & Mediastinal mass \\
\hline 7.6 & $2.41-23.91$ & $<0.001$ & $5(4.2 \%)$ & $10(25 \%)$ & Renal involvement \\
\hline 10.93 & $2.10-56.87$ & 0.001 & $2(1.7 \%)$ & $6(16.25)$ & CNS involvement \\
\hline 4.66 & $1.75-12.39$ & 0.001 & $9(8 \%)$ & $11(28.9 \%)$ & T-cell immunophenotype \\
\hline 3.88 & $1.52-9.89$ & 0.003 & $11(4.7 \%)$ & $16(19.8 \%)$ & Baseline LDH $\geq 2000 \mathrm{U} / \mathrm{L}$ \\
\hline 1.81 & $075-4.32$ & 0.18 & $18(15.1 \%)$ & $10(24.4 \%)$ & Age $\geq 10$ yr \\
\hline
\end{tabular}


Citation: Bahoush GR, Yazdi E, Ansari SH, Arjmandi KH, Vossough P (2015) Identification of Children with Acute Lymphoblastic Leukemia at Low Risk for Tumor Lysis Syndrome . J Blood Disord Transfus 6: 318. doi:10.4172/2155-9864.1000318

Page 3 of 4

\begin{tabular}{|l|l|l|l|l|l|}
\hline 1.55 & $0.76-3.17$ & 0.23 & $71(59.7 \%)$ & $20(48.8 \%)$ & Male sex \\
\hline
\end{tabular}

Table 2A: The predictors of TLS in patients with ALL (Univariate analysis).

\begin{tabular}{|l|l|l|l|}
\hline $\mathbf{P}$ value & OR & $\mathbf{9 5 \%} \mathbf{C l}$ & Variables \\
\hline 0.008 & 11.61 & $1.88-71.80$ & CNS involvement \\
\hline 0.001 & 8.07 & $2.30-28.33$ & Renal involvement \\
\hline 0.014 & 4.34 & $1.34-14.02$ & Mediastinal mass \\
\hline 0.009 & 3.11 & $1.33-7.28$ & WBC $\geq 20,000 / \mu \mathrm{L}$ \\
\hline
\end{tabular}

Table 2B: The independent predictors of TLS in multivariate logistic regression analysis.

\section{Discussion}

Tumor Lysis Syndrome (TLS) is a critical consequence of cancer therapy and delayed diagnosis could result in life threatening conditions and even patient death. So earlier recognition and appropriate treatment is crucial $[5,11]$.

It has been recommended to apply prophylactic approaches in all pediatrics or adults suffered from hematologic malignancies undergoing chemotherapy $[4,11,12]$.

In the present study about $25 \%$ of ALL patients met TLS criteria and in univariate regression model, CNS and renal involvement, T-cell immunophenotype and initial $\mathrm{WBC} \geq 20000 / \mu \mathrm{l}$ were the strongest predictors of TLS.

Truong et al in a study in Canada (2007) on 328 ALL children $(\leq 18$ years) showed that $23 \%$ met criteria for TLS. Factors predictive of TLS were male sex, age $\geq 10$ years, splenomegaly, mediastinal mass, T-cell phenotype, CNS involvement, $\mathrm{LDH} \geq 2000 \mathrm{U} / \mathrm{L}$ and $\mathrm{WBC} \geq 20 \times 10^{9} / \mathrm{L}$. The negative predictive value of developing TLS in this model was $97 \%$, with a sensitivity of $95 \%$ [13].

Beyene et al. (Canada 2009) concluded that age is the most important predictor of TLS and the second most important variable is WBC. They reported that a group at low risk of TLS consists of children younger than 10 years, without T-cell immunophenotype, whose baseline $\mathrm{WBC}$ is $<20 \times 10^{9} / \mathrm{L}$ and those with palpable spleen $<2$ $\mathrm{cm}[14]$.

In a study by Wössmann et al. on 1791 children with B-precursor ALL and stage III/IV Burkitt's lymphoma with LDH level $\geq 500 \mathrm{U} / \mathrm{L}$, TLS was reported in 78 children (4.4\%). Patients with B-precursor ALL had the highest risk to develop TLS (26.4\%) followed by Burkitt's lymphoma/leukemia and a LDH $\geq 500 \mathrm{U} / \mathrm{l}$ (14.9\%) [15].

Hesham et al. studied 60 ALL children and adolescents younger than 18-years and concluded that the strongest predictors of TLS are as followings: high intial WBC $\geq 20 \times 10 / \mathrm{L}$, followed by T-cell immunophenotyping and then initial LDH $\geq 1000 \mathrm{IU} / \mathrm{L}$, and splenomegaly. In their findings 6 cases $(10 \%)$ were at the low risk group and had no TLS [16].

Our study from a developing country identified ALL patients at low risk of developing TLS. This could result in decreased unnecessary monitoring and prevent the use of expensive drugs like Rasburicase.

\section{Declaration of Interests}

There is no source of funding for research; financial or personal relationships with other people or organizations that could inappropriately influence the work; affiliation with any organization with a financial interest, direct or indirect, in the subject matter or materials discussed in the manuscript that may affect the conduct or reporting of the work.

\section{Acknowledgement}

The authors would like to thank Farzan Institute of Research and Technology for technical assistance.

\section{References}

1. Truong TH, Beyene J, Hitzler J, Abla O, Maloney AM, et al. (2007) Features at presentation predict children with acute lymphoblastic leukemia at low risk for tumor lysis syndrome. Cancer 110: 1832-1839.

2. Pession A, Melchionda F, Castellini C (2008) Pitfalls, prevention, and treatment of hyperuricemia during tumor lysis syndrome in the era of rasburicase (recombinant urate oxidase). Biologics 2: 129.

3. Treuting PM, Albertson TM, Preston BD (2010) Case series: acute tumor lysis syndrome in mutator mice with disseminated lymphoblastic lymphoma. Toxicol Pathol 38: 476-485.

4. Tosi P, Barosi G, Lazzaro C, Liso V, Marchetti M, et al. (2008) Consensus conference on the management of tumor lysis syndrome. Haematologica 93: $1877-1885$

5. Rajendran A, Bansal D, Marwaha RK, Singhi SC (2013) Tumor lysis syndrome. Indian J Pediatr 80: 50-54.

6. Howard SC, Jones DP, Pui CH (2011) The tumor lysis syndrome. N Engl J Med 364: 1844-1854.

7. Parisi MT, Fahmy JL, Kaminsky CK, Malogolowkin MH (1999) Complications of cancer therapy in children: a radiologist's guide. Radiographics 19: 283-297.

8. Lewis MA, Hendrickson AW, Moynihan TJ (2011) Oncologic emergencies: Pathophysiology, presentation, diagnosis, and treatment. CA Cancer J Clin 61: 287-314.

9. Senbanjo IO (2009) Tumor lysis and acute renal failure in Burkitt's lymphoma: A review on pathophysiology and management. Indian J Nephrol 19: 83-86.

10. Lanzlowsky P (2005) Manual of Pediatric Hematology and Oncology. (4thedn). Elsevier Academic Press, USA: 13-14.

11. Tiu RV, Mountantonakis SE, Dunbar AJ, Schreiber MJ Jr (2007) Tumor lysis syndrome. Semin Thromb Hemost 33: 397-407.

12. Teo WY, Loh TF, Tan AM (2007) Avoiding dialysis in tumour lysis syndrome: is urate oxidase effective? - a case report and review of literature. Ann Acad Med Singapore 36: 679-683.

13. Prabhu AD, Mos K, Karl TR, Anderson B (2012) Extracorporeal life support in the acute management of tumour lysis syndrome. Interact Cardiovasc Thorac Surg 15: 568-569.

14. Beyene J, Atenafu EG, Hamid JS, To T, Sung L (2009) Determining relative importance of variables in developing and validating predictive models. BMC Med Res Methodol 9: 64.

15. Wössmann W, Schrappe M, Meyer U, Zimmermann M, Reiter A (2003) Incidence of tumor lysis syndrome in children with advanced stage Burkitt's lymphoma/leukemia before and after introduction of prophylactic use of urate oxidase. Ann Hematol 82: 160-165. 
Citation: Bahoush GR, Yazdi E, Ansari SH, Arjmandi KH, Vossough P (2015) Identification of Children with Acute Lymphoblastic Leukemia at Low Risk for Tumor Lysis Syndrome. J Blood Disord Transfus 6: 318. doi:10.4172/2155-9864.1000318

Page 4 of 4

16. Abdel-Baset HA, Eldin EN, Eltayeb AA, Hussein AM (2012) Clinical and laboratory approach for the identification of the risk for tumour lysis syndrome in children with acute lymphoblastic leukemia. Life Science Journal 9: 1 . 